\title{
Hamilton cycles, minimum degree and bipartite holes
}

\author{
Colin McDiarmid \\ cmcdQstats.ox.ac.uk
}

\author{
Nikola Yolov \\ nikola.yolov@cs.ox.ac.uk
}

April 20, 2016

\begin{abstract}
We present a tight extremal threshold for the existence of Hamilton cycles in graphs with large minimum degree and without a large "bipartite hole" (two disjoint sets of vertices with no edges between them). This result extends Dirac's classical theorem, and is related to a theorem of Chvátal and Erdős.

In detail, an $(s, t)$-bipartite-hole in a graph $G$ consists of two disjoint sets of vertices $S$ and $T$ with $|S|=s$ and $|T|=t$ such that there are no edges between $S$ and $T$; and $\widetilde{\alpha}(G)$ is the maximum integer $r$ such that $G$ contains an $(s, t)$-bipartite-hole for every pair of non-negative integers $s$ and $t$ with $s+t=r$. Our central theorem is that a graph $G$ with at least 3 vertices is Hamiltonian if its minimum degree is at least $\widetilde{\alpha}(G)$.

From the proof we obtain a polynomial time algorithm that either finds a Hamilton cycle or a large bipartite hole. The theorem also yields a condition for the existence of $k$ edge-disjoint Hamilton cycles. We see that for dense random graphs $G(n, p)$, the probability of failing to contain many edge-disjoint Hamilton cycles is $(1-p)^{(1+o(1)) n}$. Finally, we discuss the complexity of calculating and approximating $\widetilde{\alpha}(G)$.
\end{abstract}

\section{Introduction and statement of results}

Hamilton cycles are one of the central topics in graph theory, see for example BM08. The problem of recognising the existence of a Hamilton cycle in a graph is included in Karp's 21 NP-complete problems Kar72. Recall that $\delta(G)$ denotes the minimum degree $d(v)$ of a vertex $v$ in $G$. An early result by Dirac Dir52] states:

Theorem 1 (Dirac's Theorem). A graph $G$ with $n \geq 3$ vertices is Hamiltonian if $\delta(G) \geq n / 2$.

The theorem is sharp, since the disjoint union of two complete $n$-vertex graphs has minimum degree $n-1$ and it does not contain a Hamilton cycle. This example contains a large bipartite hole, that is two disjoint sets of vertices 
with no edge between them. It is natural to ask if such a hole is necessary to construct a non-Hamiltonian graph with large minimum degree. We show that indeed this is the case.

Given disjoint sets $S$ and $T$ of vertices in a graph, we let $E(S, T)$ denote the set of edges with one end in $S$ and one in $T$.

Definition 1.1. An $(s, t)$-bipartite-hole in a graph $G$ consists of two disjoint sets of vertices $S$ and $T$ with $|S|=s$ and $|T|=t$ such that $E(S, T)=\emptyset$. We define the bipartite-hole-number $\widetilde{\alpha}(G)$ to be the least integer $r$ which may be written as $r=s+t-1$ for some positive integers $s$ and $t$ such that $G$ does not contain an $(s, t)$-bipartite-hole.

An equivalent definition of $\widetilde{\alpha}(G)$ is the maximum integer $r$ such that $G$ contains an $(s, t)$-bipartite-hole for every pair of non-negative integers $s$ and $t$ with $s+t=r$. Observe that $\widetilde{\alpha}(G)=1$ if and only if $G$ is complete, and $\widetilde{\alpha}(G) \geq \alpha(G)$, where $\alpha(G)$ is the stability number of $G$. Also note that for $1 \leq a \leq b$, we have $\widetilde{\alpha}\left(K_{a, b}\right)=b$ and $\widetilde{\alpha}\left(\overline{K_{a, b}}\right)=\min \{b+1,2 a+1\}$. (Here $K_{a, b}$ denotes the complete bipartite graph with parts of sizes $a$ and $b$, and $\bar{G}$ denotes the complement of $G$.)

The following is our main theorem. It arose from our investigations of the random perfect graph $P_{n}$, where we wished to show that $P_{n}$ is Hamiltonian with failure probability $e^{-\Omega(n)}$, see MY16.

Theorem 2. A graph $G$ with at least 3 vertices is Hamiltonian if $\delta(G) \geq \widetilde{\alpha}(G)$.

This result is sharp in the sense that for every positive integer $r$ there is a non-Hamiltonian graph with $\delta(G)=r=\widetilde{\alpha}(G)-1$. An example is $G=K_{r, r+1}$, where $\delta(G)=r$ and $\widetilde{\alpha}(G)=r+1$. Theorem 2 generalises Theorem 1 of Dirac. Indeed, a graph $G$ with $\delta(G) \geq n / 2$ has no $(1,\lfloor n / 2\rfloor)$-bipartite-hole, and hence $\delta(G) \geq n / 2 \geq \widetilde{\alpha}(G)$. Also, Theorem 2 can be extended to provide a sufficient condition for the existence of many edge-disjoint Hamilton cycles; and in fact the next result will be deduced quickly from Theorem 2 .

Theorem 3. Let $r \geq 0$ be an integer, and let $G$ be a graph with at least 3 vertices such that $\delta(G) \geq(r+1) \widetilde{\alpha}(G)+3 r$. Then $G$ contains $r+1$ edge-disjoint Hamilton cycles.

Note that by setting $r=0$ in Theorem 3 we regain Theorem 2

It is perhaps not surprising that determining $\widetilde{\alpha}(G)$ is NP-hard and that it is hard to approximate, see Section 5 below. However, Theorem 2 can be made algorithmic.

Theorem 4. There is an algorithm which, on input a graph $G$ with $n \geq 3$ vertices, in $O\left(n^{3}\right)$ time outputs either a Hamilton cycle or a certificate that $\widetilde{\alpha}(G)>\delta(G)$.

Theorem 3 can also be made algorithmic. One can repeatedly use the algorithm in Theorem 4 to find a Hamilton cycle, remove its edges from $G$ and repeat, or if no cycle is found, output a certificate that $\widetilde{\alpha}(G)$ is large. This yields: 
Theorem 5. There is an algorithm that, on input a graph $G$ with $n \geq 3$ vertices, in $O\left(n^{4}\right)$ time outputs a non-negative integer $r$, a collection of $r$ edge-disjoint Hamilton cycles of $G$, and a certificate that $\widetilde{\alpha}(G)>\frac{\delta(G)-3 r}{r+1}$.

Containing a large bipartite hole is not a certificate for the absence of Hamilton cycles; there are Hamiltonian graphs for which the algorithm will stop before outputting a Hamilton cycle, which is to be expected, since deciding whether or not a graph is Hamiltonian is NP-complete.

We conclude the paper by applying Theorem 3 to show quickly that for a sufficiently dense random graph $G$, the probability of $G$ failing to contain many edge-disjoint Hamilton cycles is well-estimated by the probability that $G$ contains a vertex with too small degree $(<2 r)$, or indeed contains an isolated vertex.

Theorem 6. Let $0<\epsilon<1$, let $0 \leq p=p(n) \leq 1-\epsilon$, and let $r=r(n)$ be a positive integer. If $\frac{p(n) \sqrt{n}}{r(n) \log n} \rightarrow \infty$ as $n \rightarrow \infty$, then the probability that $G(n, p)$ fails to contain at least $r$ edge-disjoint Hamilton cycles is $(1-p)^{(1+o(1)) n}$.

Setting $r=1$ we obtain:

Corollary 7. If $p(n) \sqrt{n} / \log n \rightarrow \infty$ as $n \rightarrow \infty$, then the probability that $G(n, p)$ fails to be Hamiltonian is $(1-p)^{(1+o(1)) n}$.

\section{Related work}

Finding sufficient conditions for the existence of Hamilton cycles has been an active area of research for more than sixty years. Among the most well-known conditions are Dirac's Theorem Dir52, Theorem 1, and a generalisation by Ore Ore60, which states that an $n$-vertex graph $G$ is Hamiltonian if $d(u)+d(v) \geq n$ for any pair of non-adjacent vertices $u$ and $v$. These were further generalised by Bondy and Chvátal, and others, see the book by Bondy and Murty BM08] and see Gou03, Li13] for surveys. Both conditions are further generalised by Fan Fan84, where he proved that a 2-connected graph $G$ of order $n$ is Hamiltonian if $\max (d(u), d(v)) \geq n / 2$ for every pair of nonadjacent vertices $u, v$ with distance 2. See [DeL00] for a survey.

One of these generalisations, by Chvátal and Erdős [CE72, has a sharp condition close to the one in this paper. We denote the vertex connectivity of $G$ by $\kappa(G)$ and the number of vertices of $G$ by $v(G)$.

Theorem 8 (Chvátal-Erdős Theorem). A graph $G$ with at least 3 vertices is Hamiltonian if $\kappa(G) \geq \alpha(G)$.

There are interesting connections between Theorems 2 and 8 and between the parameters $\kappa, \delta, \alpha$ and $\widetilde{\alpha}$. For example, $\kappa(G) \leq \delta(G) \leq v(G)-\alpha(G)$ and $\alpha(G) \leq \widetilde{\alpha}(G) \leq v(G)-\kappa(G)$. Furthermore, we will see in Lemma 3.1 that $\kappa(G) \geq \delta(G)-\widetilde{\alpha}(G)+2$. 
Comparing Theorems 2 and 8, neither condition implies the other. Here is an example of a graph $G$ that meets the conditions of Theorem 2 but not Theorem [8. It has vertex set $V(G)=\{a\} \cup B \cup C \cup D$, such that $|B|=k+\ell$, $|C|=k,|D|=\ell+1$, and all these sets are disjoint. All edges between $\{a\}$ and $B$, between $B$ and $C$, and between $C$ and $D$ are present, $B$ and $D$ are complete, and $C$ is independent. It is easy to see that $k=\kappa(G)<\alpha(G)=k+1$, and $\delta(G)=k+\ell \geq \max \{k+1,2 \ell+3\}=\widetilde{\alpha}(G)$ for $\ell \geq 1$ and $k \geq \ell+3$. In the other direction, $C_{5}$ satisfies $\kappa=2=\alpha$ but $\delta=2<3=\widetilde{\alpha}$.

A more recent related result is by Hefetz, Krivelevich and Szabó [HKS09]. Roughly speaking, the authors prove that expanding graphs without large bipartite holes are Hamiltonian. Their results cover a wide range of graphs, including relatively sparse graphs. Compared to HKS09, we focus on tight extremal thresholds, simple self-contained proofs and the right conditions for edge-disjoint Hamilton cycles.

Hamilton cycles in random graphs have been well-studied, see for example [Fri89, Bol01]. In KS83] Komlós and Szemerédi prove that if

$$
p=p(n)=\frac{k(n)}{\left(\begin{array}{c}
n \\
2
\end{array}\right)} ; \quad k(n)=\frac{1}{2} n \log n+\frac{1}{2} n \log \log n+c_{n} n,
$$

then

$$
\lim _{n \rightarrow \infty} \mathbb{P}(G(n, p) \text { is Hamiltonian })= \begin{cases}0 & \text { if } c_{n} \rightarrow-\infty \\ e^{-e^{-2 c}} & \text { if } c_{n} \rightarrow c \\ 1 & \text { if } c_{n} \rightarrow \infty\end{cases}
$$

Frieze proves in Fri85 a similar result for random bipartite graphs. The evolutionary process $G_{n, t}$ is defined as follows: $G_{n, 0}$ is the empty graph on $n$ vertices and $G_{n, k+1}$ is obtained from $G_{n, k}$ by adding an edge uniformly at random. Ajtai, Komlós and Szemerédi [AKS85] and Bollobás [Bol84] showed that with high probability the hitting time for Hamiltonicity equals the hitting time for minimal degree at least two.

\section{Extremal condition for Hamilton cycle}

A necessary condition for a graph to be Hamiltonian is to be 2-connected, so Theorem 2 implies that every graph $G$ with $\delta(G) \geq \widetilde{\alpha}(G)$ is 2-connected. We give one preliminary lemma before proving Theorems 2 and 4

Lemma 3.1. The following holds for every graph $G$ :

$$
\kappa(G) \geq \delta(G)+2-\widetilde{\alpha}(G) .
$$

Proof. Suppose for a contradiction the vertices $v_{1}$ and $v_{2}$ are separated by a set $S$ of size less than $\delta(G)+2-\widetilde{\alpha}(G)$. Let $s$ and $t$ be positive integers such that $\widetilde{\alpha}(G)+1=s+t$ and $G$ has no $(s, t)$-bipartite-hole. Then $\frac{\widetilde{\alpha}(G)+1}{2} \leq \max (s, t) \leq$ $\widetilde{\alpha}(G)$. Now the closed neighbourhoods $N\left[v_{i}\right]$ satisfy $\left|N\left[v_{i}\right] \backslash S\right|^{2} \geq \delta(G)+1-|S| \geq$ 
$\widetilde{\alpha}(G) \geq \max (s, t)$. The sets $N\left[v_{1}\right] \backslash S$ and $N\left[v_{2}\right] \backslash S$ are disjoint because $S$ is a separator, but $\left|N\left[v_{1}\right] \backslash S\right| \geq s$ and $\left|N\left[v_{2}\right] \backslash S\right| \geq t$, so there is an edge between them and $S$ does not separate $v_{1}$ from $v_{2}$, a contradiction.

As an aside before proving Theorem 2, suppose the graph $G$ with at least 3 vertices satisfies $\delta(G) \geq 2 \widetilde{\alpha}(G)-2$. Then

$$
\kappa(G) \geq \delta(G)+2-\widetilde{\alpha}(G) \geq \widetilde{\alpha}(G) \geq \alpha(G) .
$$

Hence the conditions of the Chvátal-Erdős Theorem are met, and so $G$ is Hamiltonian.

Theorem 2. A graph $G$ with at least 3 vertices is Hamiltonian if $\delta(G) \geq \widetilde{\alpha}(G)$.

Proof. If $\widetilde{\alpha}(G)=1$, then $G$ is complete, and so $G$ is Hamiltonian. Thus we may suppose that $\widetilde{\alpha}(G) \geq 2$. We will show that if $P$ is a maximal length path in $G$, then $G[V(P)]$ is Hamiltonian. This, together with the connectedness of $G$ following from Lemma 3.1, is enough to complete the proof.

Indeed, suppose $P$ is a maximal length path in $G, n=v(P)$, and label the vertices in $V(P)$ with $[n]:=\{1, \ldots, n\}$ in the order they appear in the path, after choosing an arbitrary orientation. We may assume that vertices 1 and $n$ are not adjacent. For a set $S \subseteq V(P)$, define $S^{+}$to be the set of successors $x^{+}$ of elements $x$ in $S$, and define $S^{-}$to be the set of predecessors $x^{-}$. We leave $S^{+}$undefined if $n \in S$ and $S^{-}$is undefined if $1 \in S$.

We now describe three situations when $P$ can be closed to form a cycle. The first yields a standard proof of Dirac's and Ore's theorems, the second involves 'non-crossing' edges from the end vertices, and the third involves 'crossing edges'.

(a) If for some $j \in(1, n)$ we have $j \in N(1)$ and $j^{-} \in N(n)$, then $1 j-n j^{-}-1$ is a spanning cycle of $V(P)$ (where we follow the path $P$ from $j$ to $n$ and from $j^{-}$to 1$)$. See Figure 1.

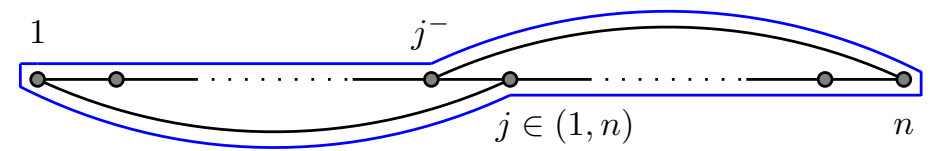

Figure 1: Single flip

(b) If for some $k \in(1, n)$ there exist $i \in N(1) \cap(1, k]$ and $j \in N(n) \cap[k, n)$ such that $i^{-}$is adjacent to $j^{+}$, then $1-i^{-} j^{+}-n j-i 1$ is a spanning cycle of $V(P)$. Here we may have $i=j$; see Figure 2 .

(c) If for some $k \in(1, n)$ there exist $i \in N(1) \cap[k, n)$ and $j \in N(n) \cap[1, k)$ such that $i^{+}$is adjacent to $j^{+}$, then $1-j n-i^{+} j^{+}-i 1$ is a spanning cycle of $V(P)$. Here we may have $j^{+}=i$; see Figure 3 . 


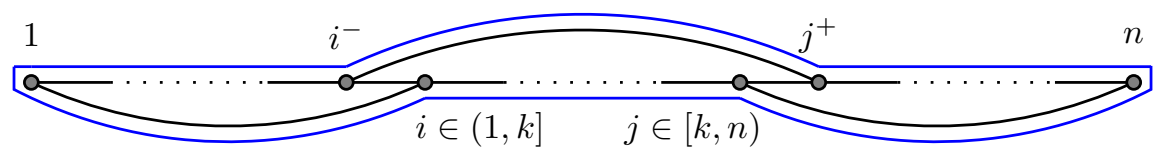

Figure 2: Double nested flip

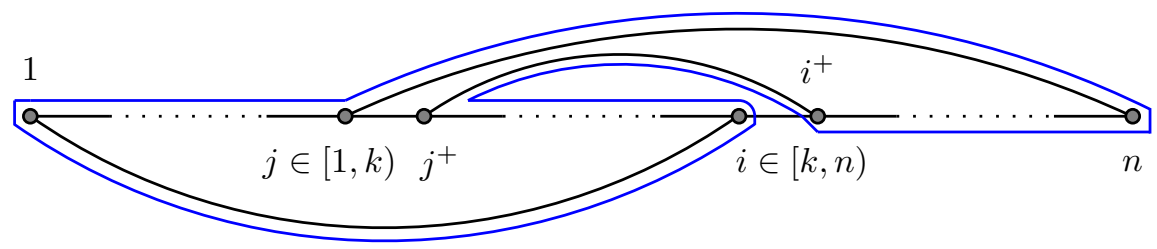

Figure 3: Double cross flip

We shall show that at least one of these situations must hold. Suppose for a contradiction that this is not the case. Then for every $k \in(1, n)$

$$
E\left[(N(1) \cap(1, k])^{-},(N(n) \cap[k, n))^{+}\right]=\emptyset
$$

since (b) does not hold; and

$$
E\left[\{1\} \cup(N(1) \cap[k, n))^{+},(N(n) \cap[1, k))^{+}\right]=\emptyset
$$

since (a) and (c) do not hold.

Let $1 \leq s \leq t$ be such that $\widetilde{\alpha}(G)+1=s+t$ and $G$ has no $(s, t)$-bipartite-hole. Since $\widetilde{\alpha}(G) \geq 2$, we have $s \leq \frac{\widetilde{\alpha}(G)+1}{2}<\widetilde{\alpha}(G)$, and hence

$$
|N(1) \cap(1,2]|=1 \leq s \leq \delta(G)-1<|N(1) \cap(1, n]|=d(1) .
$$

Therefore we can choose $k \in(1, n)$ such that $|N(1) \cap(1, k]|=s$. Equation (1) implies that $|N(n) \cap[k, n)|<t$. Since $|N(n) \cap[1, k)|+|N(n) \cap[k, n)| \geq \delta(G)$, we have $|N(n) \cap[1, k)|>\delta(G)-t \geq \widetilde{\alpha}(G)-t=s-1$, and so $|N(n) \cap[1, k)| \geq s$. Now from (2) we deduce $|N(1) \cap[k, n)|<t-1$, hence $|N(1) \cap[k, n)| \leq t-2$. Finally, since 1 is not adjacent to $n$, we have

$$
\delta(G) \leq|N(1) \cap(1, k]|+|N(1) \cap[k, n)| \leq s+t-2 \leq \delta(G)-1,
$$

and this contradiction completes the proof.

Next we consider edge-disjoint Hamilton cycles. We need a preliminary lemma. For graphs $F$ and $G$ with the same vertex set $V$, we define $F \cup G=$ $(V, E(F) \cup E(G))$ and $F-G=(V, E(F) \backslash E(G))$.

Lemma 3.2. Suppose $H_{1}, \ldots, H_{r}$ are $r \geq 1$ Hamilton cycles in a graph $G$ and let $H=H_{1} \cup \ldots \cup H_{r}$. Then $\widetilde{\alpha}(G-H)+1 \leq(r+1)(\widetilde{\alpha}(G)+1)$. 
Proof. Let $1 \leq s \leq t$ be such that $\widetilde{\alpha}(G)+1=s+t$ and $G$ has no $(s, t)$-bipartitehole. Let $U, W \subseteq V(G)$ be disjoint sets of size $s$ and $2 r s+t$ respectively. Now

$$
\left|W \backslash \Gamma_{H}(U)\right| \geq|W|-\sum_{i=1}^{r}\left|\Gamma_{H_{i}}(U)\right| \geq 2 r s+t-2 r s=t .
$$

But $G$ has no $(s, t)$-bipartite-hole, so $G-H$ has no $(s, 2 r s+t)$-bipartite-hole. Finally, we see that $\widetilde{\alpha}(G-H)+1 \leq s+2 r s+t \leq(r+1)(\widetilde{\alpha}(G)+1)$, since $s \leq \frac{\widetilde{\alpha}(G)+1}{2}$.

Theorem 3. Let $r \geq 0$ be an integer, and let $G$ be a graph with at least 3 vertices such that $\delta(G) \geq(r+1) \widetilde{\alpha}(G)+3 r$. Then $G$ contains $r+1$ edge-disjoint Hamilton cycles.

Proof. We sequentially find edge-disjoint Hamilton cycles $H_{1}, H_{2}, \ldots$. Let $0 \leq$ $i \leq r$ and suppose we have found $H_{1}, \ldots, H_{i}$. Let $G_{i}=G-\cup_{j \leq i} H_{j}$. Then by Lemma 3.2

$$
\widetilde{\alpha}\left(G_{i}\right) \leq(i+1)(\widetilde{\alpha}(G)+1)-1 \leq(r+1) \widetilde{\alpha}(G)+r \leq \delta(G)-2 r \leq \delta\left(G_{i}\right) .
$$

Hence by Theorem 2 we can find $H_{i+1}$ edge-disjoint from $H_{1}, \ldots H_{i}$.

A certificate that $\widetilde{\alpha}(G) \geq k$ consists of pairs $\left(S_{i}, T_{i}\right)$ for $i=1, \ldots,\lfloor k / 2\rfloor$ such that $S_{i}, T_{i} \subseteq V(G), S_{i} \cap T_{i}=\emptyset, E\left(S_{i}, T_{i}\right)=\emptyset$, and $\left|S_{i}\right|=i,\left|T_{i}\right|=k-i$.

Theorem 4. There is an algorithm which, on input a graph $G$ with $n \geq 3$ vertices, in $O\left(n^{3}\right)$ time outputs either a Hamilton cycle or a certificate that $\widetilde{\alpha}(G)>\delta(G)$.

Proof. First check if $G$ is connected. If not, pick two connected components and note that each has size at least $\delta(G)+1$. For each $i=1, \ldots,\lfloor(\delta(G)+1) / 2\rfloor$, any $i$ vertices from one of these components together with any $\delta(G)+2-i$ from the other form a bipartite hole, and hence we can find a certificate that $\widetilde{\alpha}(G) \geq \delta(G)+2$. So we can assume that $G$ is connected.

Maintain a path $P$ with initial length at least two. The algorithm performs at most $n$ steps, and the length of $P$ increases with each one. On each step, check if a terminal vertex of $P$ has a neighbour outside $V(P)$, and if so extend $P$. Otherwise, following the proof of Theorem 2, we can either find a sequence of bipartite holes forming a certificate as required and halt, or close $P$ to form a cycle. This cycle is either Hamiltonian and then the algorithm halts, or from the connectivity of $G$ we can attach an edge $x y$ with $x \in V(P)$ and $y \notin V(P)$ to obtain a strictly longer path starting from $y$ and spanning $V(P) \cup\{y\}$.

Each step takes $O\left(n^{2}\right)$ time, so the total time spent is $O\left(n^{3}\right)$.

\section{Application to dense random graphs}

The following result is phrased to cover the existence of one Hamilton cycle, and of many. 
Lemma 4.1. Fix $0<\epsilon<1$ and let $0 \leq p=p(n) \leq 1-\epsilon$ for all $n$. Given $r=r(n) \geq 1$, let $A_{r}$ be the event that $G(n, p)$ contains at least $r$ edge-disjoint Hamilton cycles, and let $A_{r}^{c}$ be the complementary event. Then

$$
n \log (1-p) \leq \log \mathbb{P}\left(A_{r}^{c}\right) \leq n \log (1-p)+(2+o(1)) r \sqrt{n} \log n .
$$

Proof. Let $G \sim G(n, p), t=\lceil\sqrt{n}\rceil$ and $d=r(2 t)+3 r-3$. From Theorem 3 we have $\{\widetilde{\alpha}(G) \leq 2 t\} \cap\{\delta(G) \geq d\} \subseteq A_{r}$, so

$$
\{\delta(G)=0\} \subseteq A_{r}^{c} \subseteq\{\widetilde{\alpha}(G)>2 t\} \cup\{\delta(G)<d\} .
$$

Clearly $\mathbb{P}(\delta(G)=0) \geq(1-p)^{n}=\exp (n \log (1-p))$. Also, the probability that vertex $n$ has degree at most $d-1$ is at most the expected number of $(d-1)$ subsets of $[n-1]$ such that each other vertex is not adjacent to $n$. Thus

$$
\begin{aligned}
\mathbb{P}(\delta(G)<d) & \leq n\left(\begin{array}{c}
n-1 \\
d-1
\end{array}\right)(1-p)^{(n-1)-(d-1)} \leq n^{d}(1-p)^{n} \epsilon^{-d} \\
& =\exp (n \log (1-p)+d(\log n+\log (1 / \epsilon))) .
\end{aligned}
$$

Further

$$
\begin{aligned}
\mathbb{P}(\widetilde{\alpha}(G)>2 t) & \leq \mathbb{P}(G \text { has a }(t, t) \text {-bipartite-hole }) \\
& \leq\left(\begin{array}{c}
n \\
t
\end{array}\right)^{2}(1-p)^{t^{2}} \leq\left(\frac{e n}{t}\right)^{2 t}(1-p)^{t^{2}} \leq e^{2 t} n^{t}(1-p)^{n} \\
& =\exp (n \log (1-p)+\sqrt{n}(\log n+O(1)))
\end{aligned}
$$

and the required upper bound on $\log \mathbb{P}\left(A_{r}^{c}\right)$ follows since $d=(2+o(1)) r \sqrt{n}$.

Theorem 6 and Corollary 7 follow directly from Lemma 4.1

\section{Complexity of computing and approximating $\widetilde{\alpha}(G)$}

Computing $\widetilde{\alpha}(G)$ is closely related to the following problem:

Maximum Balanced Complete Bipartite Subgraph (BCBS):

Instance: A positive integer $k$ and a bipartite graph $G$ with parts $A$ and $B$ where $|A|=|B|$;

Question: Does $G$ contain a complete bipartite graph with $k$ vertices in each part; that is, does $G$ have a subgraph $K_{k, k}$ ?

We use lemma 2.2 of $\mathrm{ADL}^{+94}$. By that result the BCBS problem is NPcomplete. Also, BCBS is problem [GT24] in [GJ79.

Bipartite Hole-Number (BHN):

Instance: A positive integer $k$ and a graph $G$;

Question: Is $\widetilde{\alpha}(G) \geq k$ ?

To compare the two problems we introduce the following lemma: 
Lemma 5.1. Given a graph $G$ and an integer $k$, let $G^{\prime}$ be formed from $G$ by adding a disjoint copy of $K_{k-1,2 k}$; and let $G_{k}^{\phi}$ be the complement of $G^{\prime}$. Then $K_{k, k} \subseteq G$ if and only if $\widetilde{\alpha}\left(G_{k}^{\phi}\right) \geq 2 k$.

Proof. We see that $G_{k}^{\phi}$ has an induced copy of $K_{k-1} \cup K_{2 k}$, and so it has an $(s, 2 k-s)$-bipartite-hole for each $s=1, \ldots, k-1$. Thus $\widetilde{\alpha}\left(G_{k}^{\phi}\right) \geq 2 k$ if and only if $G_{k}^{\phi}$ has a $(k, k)$-bipartite-hole; and that happens if and only if the complement of $G$ has a $(k, k)$-bipartite-hole, if and only if $G$ has a subgraph $K_{k, k}$.

The next proposition follows as a corollary.

Proposition 5.2. The BHN problem is NP-complete.

In fact, a stronger statement could be given: the BHN problem is hard to approximate. To this end we use a result from FK04 stating that the BCBS problem cannot be approximated within a factor of $2^{(\log n)^{\delta}}$ for some $\delta>0$, unless 3-SAT can be solved in $O\left(2^{n^{3 / 4+\epsilon}}\right)$ time for every $\epsilon>0$. The widely believed Exponential Time Hypothesis (ETH) states that 3-SAT cannot be solved in $2^{o(n)}$ time, which provides strong evidence for the inapproximability of BCBS. Lemma 5.1 allows us to directly translate these results to hardness of approximating $\widetilde{\alpha}(G)$ :

Proposition 5.3. There exists $\delta>0$ such that $\widetilde{\alpha}(G)$ cannot be approximated within a factor of $2^{(\log n)^{\delta}}$ provided that $3-S A T \notin D T I M E\left(2^{n^{3 / 4+\epsilon}}\right)$ for some $\epsilon>0$.

\section{Concluding remarks}

In this paper we presented a tight sufficient condition for Hamiltonicity, Theorem 2. and used that result to prove an extension concerning the existence of $r$ edge-disjoint Hamilton cycles, Theorem 3, As an application of these theorems, we proved results on disjoint Hamilton cycles in dense random graphs. It was pointed out to one of us by Michael Krivelevich that results from [HKS09] should allow us to extend Corollary 7 to much lower edge-probabilities $p(n)$, down to near the threshold for the necessary minimum degree; and indeed this is the case as long as $\frac{n p(n) \log \log \log \log n}{\log n \log \log \log n} \rightarrow \infty$, see the appendix in arXiv:math.CO/1604.00888.

We are not aware of any examples where the inequality in Theorem [3] is sharp for $r \geq 1$. It would be interesting to find such examples or relax the condition. 


\section{Appendix: Note on the probability of containing a Hamilton cycle around the threshold}

The external neighbourhood of a set $S \subseteq V$ is denoted by $N(S)$, that is

$$
N(S)=\{v \in V \backslash S: v \text { is adjacent to some } u \in S\} .
$$

We use the following result by Hefetz et al. [HKS09.

Theorem 9. Suppose $12 \leq d \leq e^{\sqrt[3]{\log n}}$ and $m=m(n, d)=\frac{\log n \log \log \log n}{\log d \log \log n}$. If $G=(V, E)$ is a graph such that

P1 for each $S \subseteq V$ if $|S| \leq \frac{n}{d m}$, then $|N(S)| \geq d|S|$, and

P2 for every disjoint $A, B \subseteq V$ if $|A|,|B| \geq \frac{n}{4130 m}$, then $E(A, B) \neq \emptyset$,

then $G$ is Hamiltonian for large $n$.

Lemma 6.1. If $p \geq 16520 \frac{m \log (e 4130 m)}{n}$, then $G(n, p)$ fails to satisfy P2 with probability less than $e^{-2 n p}$ for large $n$.

Proof. It is sufficient to show that the claim holds for each $|A|$ and $|B|$ of size $k=\left\lceil\frac{n}{4130 m}\right\rceil$. The number of choices for $A, B$ is at most

$$
\left(\begin{array}{l}
n \\
k
\end{array}\right)^{2} \leq\left(\frac{e n}{k}\right)^{2 k}=\exp \left(2 k \log \frac{e n}{k}\right) .
$$

The probability that a fixed pair $(A, B)$ is edgeless is $(1-p)^{k^{2}} \leq \exp \left(-p k^{2}\right)$. Now use the union bound to deduce that the log of the probability that P2 fails is at most

$$
2 k \log \frac{e n}{k}-p k^{2} \leq-\frac{1}{2} p k^{2} \leq-2 p n,
$$

where the first inequality holds since $p \geq \frac{4 \log (e 4130 m)}{\frac{n}{4130 m}} \geq \frac{4 \log \frac{e n}{k}}{k}$, and the second holds for large $n$.

Lemma 6.2. If $p \geq(8 d+12) \frac{\log n}{n}$, then $G(n, p)$ fails to satisfy P1 for some $|S| \geq 2$ with probability at most $e^{-\frac{3}{2} p n}$ for large $n$.

Proof. The probability that a set $S$ of size $s, 2 \leq s \leq \frac{n}{d m}$, is not expanding is at most

$$
p_{0}=\left(\begin{array}{c}
n \\
s
\end{array}\right)\left(\begin{array}{c}
n-s \\
\lfloor d s\rfloor
\end{array}\right)(1-p)^{s(n-s-d s)} \leq \exp ((d+1) s \log n) \exp \left(-\frac{7}{8} n s p\right),
$$

since $n-s-d s \geq \frac{7}{8} n$ for large $n$. Note that $\frac{a x+b}{c x+d}$ is increasing in $x$ on $\left(-\frac{d}{c}, \infty\right)$ iff $a d-c b>0$ and decreasing on $\left(-\frac{d}{c}, \infty\right)$ otherwise, hence $\frac{(d+1) s+1}{\frac{7}{8} s-\frac{3}{2}}$ is decreasing 
in $s$ on $\left(\frac{12}{7}, \infty\right)$, and therefore $\frac{(d+1) s+1}{\frac{7}{8} s-\frac{3}{2}} \leq 8 d+12$, since $s \geq 2$. Finally,

$$
\begin{aligned}
& p n \geq(8 d+12) \log n \geq \frac{(d+1) s+1}{\frac{7}{8} s-\frac{3}{2}} \log n \\
\Rightarrow & \log n+(d+1) s \log n-\frac{7}{8} n s p \leq-\frac{3}{2} p n \\
\Rightarrow & n p_{0} \leq \exp \left(-\frac{3}{2} p n\right) .
\end{aligned}
$$

Taking the union bound over all $s \geq 2$ yields the statement.

We see that the failure probability is dominated by the probability of a single low-degree vertex.

Lemma 6.3. Suppose $\frac{d \log n}{n}<<p<\frac{1}{2}$. Then

$$
\mathbb{P}(\delta(G(n, p)) \leq d) \leq \exp (-p n(1+o(1))) .
$$

Proof.

$$
\begin{aligned}
\mathbb{P}(\delta(G)<d) & \leq n\left(\begin{array}{c}
n-1 \\
d-1
\end{array}\right)(1-p)^{(n-1)-(d-1)} \leq n^{d} e^{-p n} 2^{d} \\
& =\exp (-p n+d \log (2 n))=\exp (-p n(1+o(1))) .
\end{aligned}
$$

For $k \geq 1$ the notation $\log ^{(k)} n$ stands for $\log n$ if $k=1$ and $\log \left(\log { }^{(k-1)} n\right)$ otherwise. To wrap things up, note that $\log m=\Theta\left(\log ^{(2)} n\right)$, hence $m \log m=$ $\Theta\left(\frac{\log n \log (3)}{\log d}\right)$. By solving the equation $d \log n=m \log m$ we get $d \log d=$ $\Theta\left(\log ^{(3)} n\right)$ and hence $d=\Theta\left(\frac{\log ^{(3)} n}{\log ^{(4)} n}\right)$. Therefore if $p=\frac{\log n \log ^{(3)} n}{n \log ^{(4)} n} \omega(1)$, the probability of $G(n, p)$ failing to contain a Hamilton cycle is $e^{-n p(1+o(1))}$.

\section{References}

[ADL $\left.{ }^{+} 94\right]$ Noga Alon, Richard A Duke, Hanno Lefmann, Vojtech Rodl, and Raphael Yuster. The algorithmic aspects of the regularity lemma. Journal of Algorithms, 16(1):80-109, 1994.

[AKS85] Miklós Ajtai, János Komlós, and Endre Szemerédi. First occurrence of hamilton cycles in random graphs. North-Holland Mathematics Studies, 115:173-178, 1985.

[BM08] J.A. Bondy and U.S.R. Murty. Graph Theory. Springer, 2008.

[Bol84] Béla Bollobás. The evolution of sparse graphs Graph Theory and Combinatorics.(Proc. Cambridge Combinatorics Conference in Honour of Paul Erdos). 1984. 
[Bol01] Béla Bollobás. Graph Theory, 2nd edition. Cambridge University Press, 2001.

[CE72] Vašek Chvátal and Paul Erdős. A note on hamiltonian circuits. Discrete Mathematics, 2(2):111-113, 1972.

[DeL00] Melissa DeLeon. A study of sufficient conditions for hamiltonian cycles. Department of Mathematics and Computer Science, Seton Hall University, 2000.

[Dir52] Gabriel Andrew Dirac. Some theorems on abstract graphs. Proceedings of the London Mathematical Society, 3(1):69-81, 1952.

[Fan84] Geng-Hua Fan. New sufficient conditions for cycles in graphs. Journal of Combinatorial Theory, Series B, 37(3):221-227, 1984.

[FK04] Uriel Feige and Shimon Kogan. Hardness of approximation of the balanced complete bipartite subgraph problem. Dept. Comput. Sci. Appl. Math., Weizmann Inst. Sci., Rehovot, Israel, Tech. Rep. MCS04-04, 2004.

[Fri85] A.M. Frieze. Limit distribution for the existence of hamiltonian cycles in random bipartite graphs. European Journal of Combinatorics, 6:327 - 334, 1985 .

[Fri89] A.M. Frieze. On matchings and hamilton cycles in random graphs. Lond. Math. Soc. Lect. Notes Ser., 141:84-114, 1989.

[GJ79] Michael Garey and David Johnson. Computers and intractability: A guide to the theory of NP-completeness. WH Freeman \&3 Co., San Francisco, 1979.

[Gou03] R.J. Gould. Advances on the hamiltonian problem - a survey. Graphs and Combinatorics, 19(1):7-52, 2003.

[HKS09] Dan Hefetz, Michael Krivelevich, and Tibor Szabó. Hamilton cycles in highly connected and expanding graphs. Combinatorica, 29(5):547-568, 2009.

[Kar72] Richard M Karp. Reducibility among combinatorial problems. Springer, 1972.

[KS83] János Komlós and Endre Szemerédi. Limit distribution for the existence of hamiltonian cycles in a random graph. Discrete Mathematics, 43(1):55-63, 1983.

[Li13] Hao Li. Generalizations of Diracs theorem in hamiltonian graph theorya survey. Discrete Mathematics, 313(1):2034-2053, 2013.

[MY16] Colin McDiarmid and Nikola Yolov. Random perfect graphs. In preparation, $2016+$. 
[Ore60] Oystein Ore. Note on hamilton circuits. The American Mathematical Monthly, 67(1):p. 55, 1960. 